www.jmscr.igmpublication.org

Impact Factor 5.84

Index Copernicus Value: 71.58

ISSN (e)-2347-176x ISSN (p) 2455-0450

crossref DOI: _https://dx.doi.org/10.18535/jmscr/v5i9.113

Journal Of Medical Science And Clinical Research

\title{
A Rare Case of Midgut Volvulus in a 14 Yr Young Girl
}

\author{
Authors \\ Prof Dr Sribatsa Kumar Mahapatra ${ }^{1}$, Asst. Prof Dr Debashisha Roy ${ }^{2}$ \\ Dr Dharbind Kumar Jha ${ }^{3}$ \\ ${ }^{1}$ Unit Head and Professor, ${ }^{2}$ Assistant Professor, ${ }^{3}$ Post Graduate Student \\ Department of General Surgery, VIMSAR, Burla, Odisha, India \\ Contact number 9437025626, Email: drskm2010@gmail.com
}

\begin{abstract}
Intestinal malrotation is diagnosed in the first month. After birth for the great majority of patients. The incidence of malrotation in adulthood is $0.2 \%$. A 14-year girl presented to our emergency department with c/o bilious vomiting for last 5days, pain abdomen in periumbillical region for last 3 days and not passing stool for last 3 days. No h/o fever or burning micturation. midgut malrotation was diagnosed in ultrasonography and CT scan. Then exploratory laparotomy followed by the division of ladd band done. Derotation of malrotated midgut done. Appendicectomy done. placement of illeocaecal junction to left iliac fossa and jejunum on right paracolic gutter done. Peritoneal toileting done and abdomen was closed in layers. Post operatively patient recovered well. Malrotation of gut in adult with features of acute intestinal obstruction is rare Complete resolution of acute obstruction or chronic abdominal pain is the result of a high index of suspicion for malrotation, appropriate diagnostic studies and aggressive definitive treatment.

Key Words: Malrotation, Adult, Midgut volvulus, Ladd.
\end{abstract}

\section{Introduction}

Intestinal malrotation is diagnosed in the first month after birth for the great majority of patients. The incidence of malrotation in adulthood is $0.2 \%^{[1,2]}$. Four potential anomalies may occur: nonrotation, malrotation, reverse rotation, and paraduodenal hernia, with malrotation of the midgut being the most common. Anatomic variation is common in patients with intestinal malrotation and may include an abnormally high position of the cecum and a narrow mesenteric pedicle, which can lead to midgut volvulus and intestinal ischemia. In the absence of volvulus, congenital adhesive bands (Ladd bands) may compress the duodenum and cause chronic obstruction. Most adult cases are silent throughout life and are discovered incidentally unless they cause acute or chronic abdominal pain. The diagnosis for adult patients therefore relies on a high degree of clinical suspicion that may be confirmed by appropriate radiologic studies. ${ }^{[3]}$ we are going to present a rare case of small gut volvulus due to malrotation in a $14 \mathrm{yr}$ girl.

\section{Case Report}

A 14-year girl presented to our emergency department with c/o bilious vomiting for last 5days, pain abdomen in periumbillical region for 


\section{JMSCR Vol||05||Issue||09||Page 28050-28053||September}

last 3 days and not passing stool for last 3 days. No h/o fever or burning micturation. The patient had attended menarche at $13 \mathrm{yrs}$ of age and LMP was 7 days back.

O/E patient was conscious, oriented, BP 100/60 mm of Hg, PR 110/Min, per abdominal examination revealed mild distended abdomen. Soft, diffuse tenderness more at right iliac fossa, no free fluid present. Bowel sound was sluggish. DRE normal. Hemoglobin, white blood cell count and basic chemistry panel were all within normal values. Abdominal x-rays revealed two air fluid levels in the right abdomen and air within the colon. Usg findings were the Superior mesenteric artery is located in right side of superior mesenteric vein. Bowel loops peristalsis normal. Minimal anechoic collection noted in peritoneal cavity in between bowel loops suggesting midgut malrotation with minimal ascites. She was admitted and managed with nasogastric tube decompression and intravenous fluid hydration under continuous re-evaluation. CECT finding was oral contrast filled stomach and duodenum are mildly distended. Twisting of mesentry (at midline- right paramedian location) with inverse location of superior mesenteric artery and vein noted at level of L3 vertebral body. Calcification noted along the root of the mesntry over a length of $1 \mathrm{~cm}$. Small bowel loops are located in the right lumbar region and iliac fossa with displacement of the caecum. Descending colon and rectum are normally situated. No retroperitoneal lymphadenopathy or ascites seen. Suggesting small intestinal volvulus with malrotation [fig 1, 2]. Thus we planned for exploratory laparotomy. Intraoperatively we got small gut found in right lumbar and iliac fossa with displacement of ileocaecum to right hypochondrium. Ladd band from retroperitoneum compressing over the duodenum. Dilated superior mesenteric vessels found and it was malrotated. Stomach, esophagus, spleen, Gall bladder are normal. The division of ladd band done[fig 3,4,5]. Derotation of malrotated midgut done. Appendicectomy done. placement of illeocaecal junction to left iliac fossa and jejunum on right paracolic gutter done. Peritoneal toileting done and abdomen was closed in layers.post operatively patient recovered well.

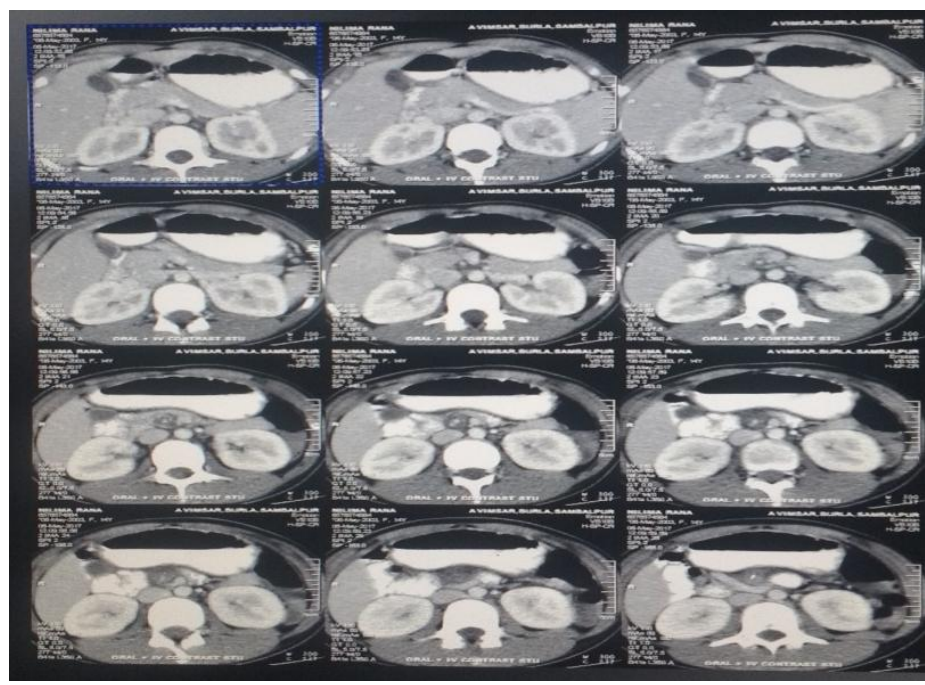

Fig 1: Showing CT Scan of Abdomen with Features of Malrotated Midgut

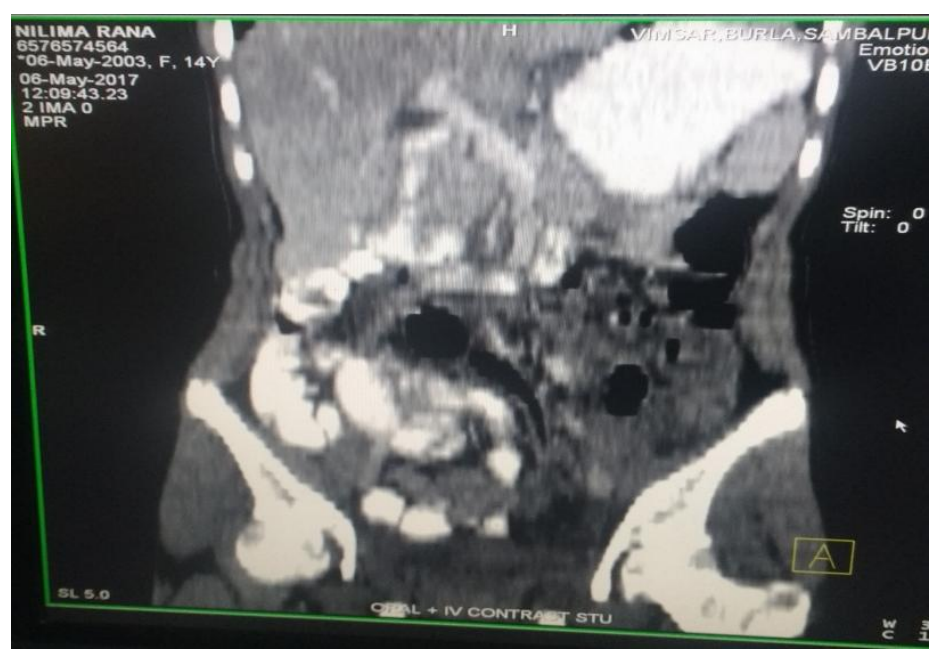

Fig 2: Showing Saggital View of CT of Abdomen with Features of Malrotated Midgut

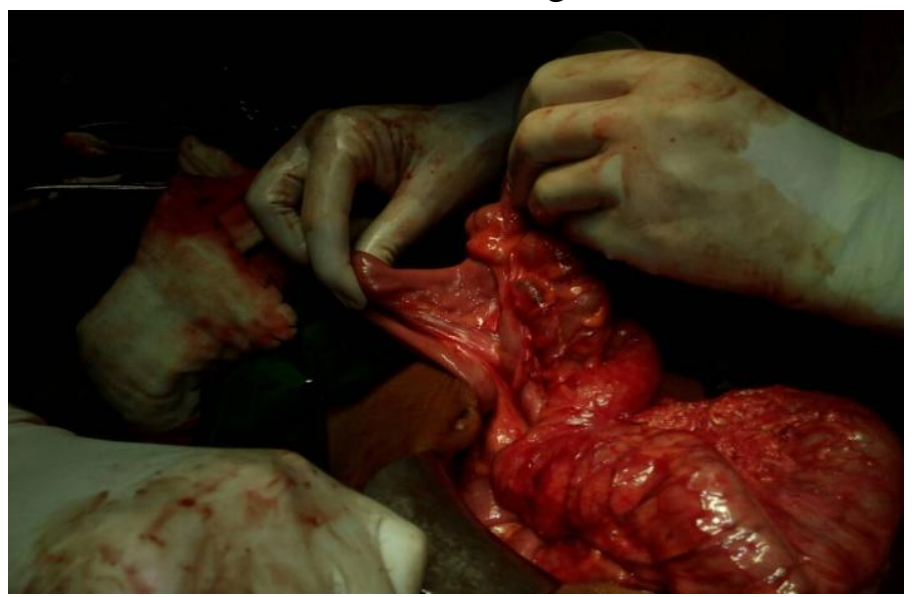

Fig 3: Showing Ileocaecal Junction in Right Hypochondrium with Malrotation 


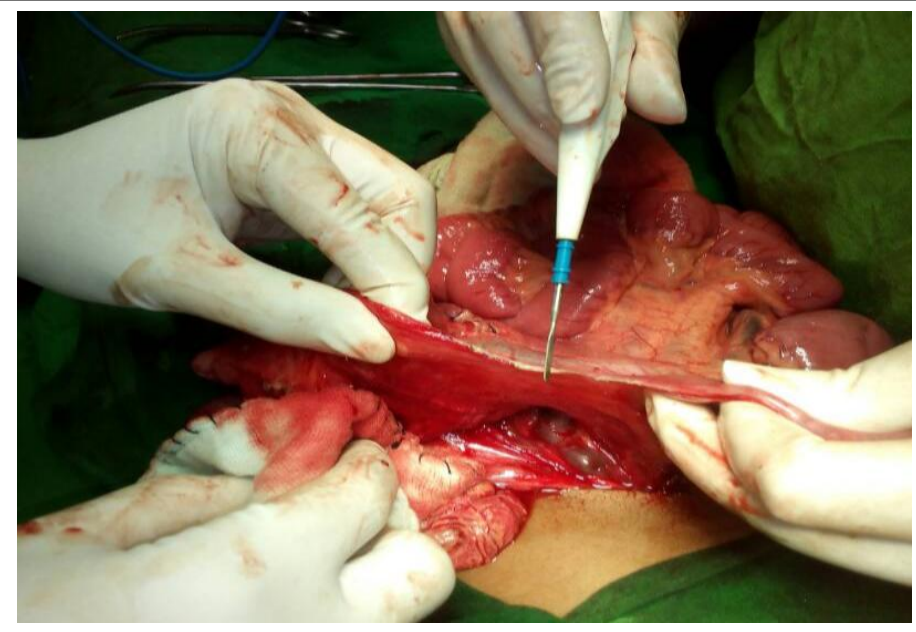

Fig 4: Division of Ladd Band

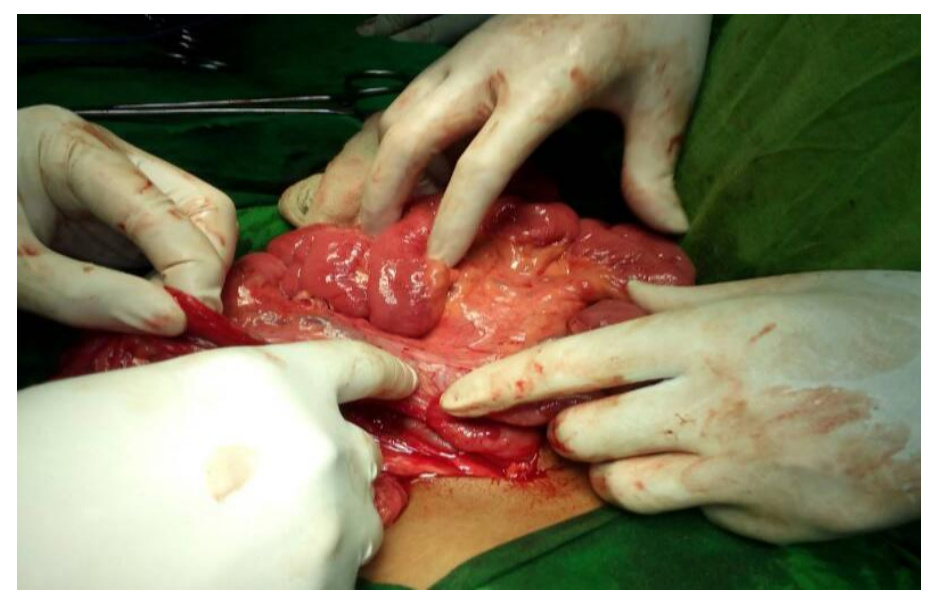

Fig 5: Midgut Derotated

\section{Discussion}

Intestinal malrotion with complications is often seen in infants and children, but few cases have been reported in adults ${ }^{[3-5]}$. Most often $(60 \%-$ $85 \%)$ the condition is diagnosed in the first month of lif ${ }^{[3-5]}$ Most adult cases are silent throughout life and are discovered incidentally unless they cause acute or chronic abdominal pain. Many patients have ill-defined abdominal complaints from childhood. During embryonic development, the midgut herniates into the umbilical sac. At 4 week's gestation, the embryo's gut is in the form of a straight tube. During the fifth week, a loop develops with the superior mesenteric artery (SMA) at its axis. The SMA supplies blood to the midgut. Intestinal rotation primarily involves the midgut. The rotation involves three stages. The first stage occurs in weeks 5-10. It is a 90-degree counterclockwise rotation of the midgut, and the bowel returns to the abdominal cavity. The second stage occurs at week 11 and involves a further 270-degree counter clockwise rotation within the abdominal cavity so that the duodenal " $\mathrm{C}$ "' loop and small bowel become attached to the posterior abdominal wall with the ascending colon to the right, the transverse colon above, and descending colon to the left. In the third stage, the cecum descends, and the ascending and descending colon attach to the posterior abdomen, with fusion and anchoring of the mesentery ${ }^{[2,4]}$. Disturbances during any of the individual stages result in anomalies of rotation and abnormalities of mesenteric fixation. Intestinal malrotation can cause shortness of the mesenteric root and narrowness of the resulting suspensory pedicle of the gut. The shortened mesentery allows the small bowel to twist around the narrowed pedicle of the superior mesenteric artery, which places patient at high risk of volvulus of the midgut and intestinal ischemia. Even though there is no volvulus, congenital adhesive bands (Ladd's bands) may compress the duodenum and cause chronic obstruction $^{[5]}$. The spectrum of abnormalities can be classified into various subtypes based on the stage of midgut. But for practical purposes, it is useful to simplify Categorization into nonrotation and incomplete rotation. "Nonrotation" is the "classic" form of malrotation, it represents most cases identified in adults ${ }^{[4]}$. Unlike the pediatric population, most adult patients with malrotation lack other congenital anomalies and are free of symptoms related to their malrotated bowel ${ }^{[11]}$. The few adults who do have symptoms typically present with a prolonged history of abdominal complaints suggestive of obstruction. Symptoms in the adult patient are often mistaken for irritable bowel syndrome, peptic ulcer disease, biliary and pancreatic disease, and psychiatric disorders. In general, there are three distinct patterns of presentation of malrotation in adults ${ }^{[5]}$.

The first group of patients will present with acute obstructive symptoms. The second group will present with chronic symptoms of bowel obstruction. The third group will present with atypical symptoms from a common abdominal disease. 
Computed tomography (CT) is used increasingly today.Malrotation can be diagnosed on CT by a right-sided small bowel, a left-sided cecum, an inverse relationship between SMA and the superior mensenteric vein (SMV), and

aplasia of the uncinate process ${ }^{[12]}$. The inverse positions of SMV in the setting of malrotation was first described by Nichols and Li. It refers to the superior mesenteric vein lying to the left of the SMA instead of to the right ${ }^{[16]}$. A distinctive whirlpool-like pattern on CT due to encircling of the loops of the bowel around the SMA was first described by Fisher in a patient with midgut volvulus $^{[17]}$. Intestinal malrotation can cause shortness of the mesenteric root and narrowness of the resulting suspensory pedicle of the gut. The shortened mesentery allows the small bowel and mesentery to twist around the narrowed pedicle of SMA with midgut volvulus, which may be seen on $\mathrm{CT}$ as the so-called whirlpool sign.

Ultrasonography has also been reported as a diagnostic tool in intestinal malrotation. Characteristic signs of midgut volvulus include duodenal dilation with distal tapering, fixed midline bowel, the whirlpool sign ${ }^{[18]}$, and dilation of the SMV. It also allows an accurate estimate of the number of twists the mesentery has undergone, which may be helpful when the surgeon attempts to reduce them at operation ${ }^{[19]}$. In addition, the relative positions of the SMA and SMV can indicate malrotation even in the absence of volvulus. Because abdominal ultrasonography and CT can readily demonstrate the relative positions of the SMA and SMV, they are valuable tools for diagnosing midgut malrotation and volvulus.

The classical treatment for intestinal malrotation is the Ladd procedure, which entails counter clockwise detorsion of the midgut volvulus (if present), division of the abnormal coloduodenal Ladd's bands tethering the midgut and causing extrinsic compression, mobilization of the duodenum, division of adhesions around the SMA to broaden the mesenteric base to prevent further volvulus, and an appendectomy.

\section{Conclusion}

Malrotation of gut in adult with features of acute intestinal obstruction is rare. Complete resolution of acute obstruction or chronic abdominal pain is the result of a high index of suspicion for malrotation, appropriate diagnostic studies and aggressive definitive treatment.

\section{References}

1. Frantzides CT, Cziperle DJ, Soergel K, Stewart E (1996) Laparoscopic Ladd procedure and cecopexy in the treatment of malrotation beyond the neonatal period. Laparosc Endosc Percutan Tech 6: 73-75

2. Mazziotti MV, Strassberg SM, Langer JC (1997) August. Intestinal rotation abnormalities without volvulus: the role of laparoscopy. J Am Coll Surg 185: 172176

3. Matzke GM, Dozois EJ, Larson DW, Moir CR. Surgical management of intestinal malrotation in adults: comparative results for open and laparoscopic Ladd procedures. Surg Endosc 2005;19:1416-1419

4. McIntosh R, Donovan EJ (1939) Disturbances of rotation of the intestinal tract. Am J Dis Child 57:116-166

5. Kiesewetter WB, Smith JW (1958) Malrotation of midgut in infancy and childhood. AMA Arch Surg 77:483-491. 